\title{
The influence of resistivity gradients on shock conditions for a Petschek reconnection geometry
}

\author{
Christian Nabert $^{1}$ and Karl-Heinz Glassmeier ${ }^{1,2}$ \\ ${ }^{1}$ Institut für Geophysik und extraterrestrische Physik, Technische Universität Braunschweig, Germany \\ ${ }^{2}$ Max-Planck-Institut für Sonnensystemforschung, Göttingen, Germany \\ Correspondence to: Christian Nabert (c.nabert@tu-bs.de)
}

Received: 18 February 2016 - Accepted: 4 April 2016 - Published: 13 April 2016

\begin{abstract}
Shock waves can strongly influence magnetic reconnection as seen by the slow shocks attached to the diffusion region in Petschek reconnection. We derive necessary conditions for such shocks in a nonuniform resistive magnetohydrodynamic plasma and discuss them with respect to the slow shocks in Petschek reconnection. Expressions for the spatial variation of the velocity and the magnetic field are derived by rearranging terms of the resistive magnetohydrodynamic equations without solving them. These expressions contain removable singularities if the flow velocity of the plasma equals a certain characteristic velocity depending on the other flow quantities. Such a singularity can be related to the strong spatial variations across a shock. In contrast to the analysis of Rankine-Hugoniot relations, the investigation of these singularities allows us to take the finite resistivity into account. Starting from considering perpendicular shocks in a simplified one-dimensional geometry to introduce the approach, shock conditions for a more general two-dimensional situation are derived. Then the latter relations are limited to an incompressible plasma to consider the subcritical slow shocks of Petschek reconnection. A gradient of the resistivity significantly modifies the characteristic velocity of wave propagation. The corresponding relations show that a gradient of the resistivity can lower the characteristic Alfvén velocity to an effective Alfvén velocity. This can strongly impact the conditions for shocks in a Petschek reconnection geometry.
\end{abstract}

Keywords. Space plasma physics (magnetic reconnection; shock waves)

\section{Introduction}

In space plasmas, one of the most efficient conversion mechanisms of magnetic energy into kinetic or thermal energy is magnetic reconnection. Reconnecting magnetic field lines change the magnetic field topology due to a finite electrical resistivity and the plasma can be highly accelerated as described by the Sweet-Parker model (Sweet, 1958; Parker, 1957). Petschek (1964) claimed that it is not only diffusion that can convert magnetic energy into kinetic but that additional slow shocks can also do so. Such shocks attached to a resistive region can significantly modify the reconnection geometry and enhance the reconnection rate. Plasma simulations of magnetic reconnection show that a nonuniform resistivity profile with a strong locally enhanced resistivity lead to a reconnection solution with slow shocks (e.g., Ugai and Tsuda, 1977; Scholer, 1989; Yan et al., 1992). Such a locally enhanced resistivity can be caused by, e.g., microinstabilities (see, e.g., Treumann, 2001).

A nonuniform resistivity can significantly impact the magnetohydrodynamic (MHD) flow by the occurrence of shocks. For an ideal MHD plasma, the Rankine-Hugoniot relations demonstrate that a flow velocity which exceeds the magnetosonic velocity can lead to shocks (see, e.g., Petrinec and Russell, 1997). Considering shock transitions with a finite thickness, as expected for resistive MHD, requires a different approach. We derive expressions for the spatial velocity and magnetic field variation by rearranging terms of the resistive MHD equations. Under certain conditions, removable singularities of these expressions can be related to the strong spatial variations of the flow quantities at shocks. A related approach was applied to a one-dimensional hydrodynamic situation to examine the influence of cosmic rays on the crit- 
ical Mach number (e.g., Achterberg, 1987; Ko et al., 1997; Becker and Kazanas, 2001). Burgess and Scholer (2015) considered such an approach for the two fluid MHD equations to discuss the dissipation mechanisms of sub- and supercritical shocks for a uniform resistive plasma. However, in the expressions derived in these approaches, the spatial variations across a shock can be caused by terms in the numerator as well as by singularities due to the denominator. In contrast, the occurrence of shocks in our expressions is restricted to the singularities. Thus, the singularities determine necessary conditions for shocks. Here, the results are discussed for the incompressible limit of Petschek reconnection, which is related to subcritical slow shocks.

\section{Quasi one-dimensional geometry}

To introduce the procedure of how the velocity conditions for shocks can be derived from the resistive and nonuniform MHD equations, a quasi one-dimensional situation is considered. The magnetic field is restricted to the $x$ direction, i.e., $\boldsymbol{B}=\left(B_{x}, 0,0\right)^{T}$. All quantities can vary with $z$ only. The flow velocity is along the $z$ direction, i.e., $\boldsymbol{u}=\left(0,0, u_{z}\right)^{T}$. The only exception is that a flow divergence may be present so that the flow can be deflected $\left(\partial_{x} u_{x} \neq 0, \partial_{y} u_{y} \neq 0\right)$. Note that this situation often is a good approximation for stagnation streamline flows. Then the stationary continuity equation $(\nabla \cdot(\rho \boldsymbol{u})=0)$ with the mass density $\rho$ can be written as

$\partial_{z} \rho=-\frac{\rho\left(\partial_{x} u_{x}+\partial_{y} u_{y}+\partial_{z} u_{z}\right)}{u_{z}}$.

The stationary induction equation for a plasma with nonuniform resistivity $\eta$ is

$\nabla \times(\boldsymbol{u} \times \boldsymbol{B})=\nabla \times(\eta \boldsymbol{j})$,

where $\boldsymbol{j}=\nabla \times \mathbf{B} / \mu_{0}$ is the current density. To simplify the representation, we set $\mu_{0}=1$ in the following. The induction equation is solved with respect to $\partial_{z} B_{x}$, which gives

$\partial_{z} B_{x}=\frac{-B_{x}\left(\partial_{z} u_{z}+\partial_{y} u_{y}\right)+\eta \partial_{z}^{2} B_{x}}{u_{z}-\partial_{z} \eta}$.

Note that the denominator is the result of a modification of the advective contribution $u_{z} \partial_{z} B_{x}$ due to the term $\left(\partial_{z} \eta\right) \partial_{z} B_{x}$ in the induction equation (Zita, 2013). The stationary momentum equation of MHD is

$\rho(\boldsymbol{u} \cdot \nabla) \boldsymbol{u}+\nabla p-\boldsymbol{j} \times \boldsymbol{B}=0$,

with the gas pressure $p$. The spatial variation of the gas pressure is substituted by $\partial_{z} p=c_{\mathrm{S}}^{2} \partial_{z} \rho$, with the sound speed $c_{\mathrm{S}}:=\left(\partial_{\rho} p\right)^{0.5}$. Substituting Eqs. (1) and (3) in the momentum Eq. (4) and solving the momentum equation with respect to $\partial_{z} u_{z}$ yields

$\partial_{z} u_{z}=\frac{c_{\mathrm{S}}^{2} D_{\mathrm{S}}+\kappa u_{\mathrm{A}, x}^{2} D_{\mathrm{S} 1}}{u_{z}^{2}-c_{\mathrm{S}}^{2}-\kappa u_{\mathrm{A}, x}^{2}}$, where $u_{\mathrm{A}, x}$ is the $x$ component of the Alfvén velocity $\mathbf{u}_{\mathrm{A}}:=$ $\boldsymbol{B} / \rho^{0.5}$ and $\kappa$ is defined by

$\kappa:=u_{z} /\left(u_{z}-\partial_{z} \eta\right)$.

Further, we substituted $D_{\mathrm{S}}:=\partial_{y} u_{y}+\partial_{x} u_{x}$ and $D_{\mathrm{A} 1}:=$ $\partial_{y} u_{y}-\left(\eta \partial_{z}^{2} B_{x}\right) / B_{x}$, terms corresponding to flow modifications, e.g., due to an obstacle in the flow. In Eq. (5), the Alfvén velocity $u_{\mathrm{A}, x}$ is scaled by $\kappa^{0.5}$. Therefore, we introduce an effective Alfvén velocity $\widetilde{u}_{\mathrm{A}, x}$, defined by

$\widetilde{u}_{\mathrm{A}, x}:=\kappa^{0.5} u_{\mathrm{A}, x}$.

The modification of the Alfvén velocity $u_{\mathrm{A}, x}$ is due to a resistivity gradient, and for $\partial_{z} \eta \rightarrow 0$, the effective Alfvén velocity $\tilde{u}_{\mathrm{A}, x}$ equals the Alfvén velocity $u_{\mathrm{A}, x}$.

Across a shock, the MHD quantities, e.g., the flow velocity, change their values. We restrict our analysis to approximately normal shocks, i.e., the upstream flow velocity is approximately orthogonal to the shock. Thus, $\partial_{x} u_{x}$ and $\partial_{y} u_{y}$ are usually small compared to $\partial_{z} u_{z}$ and $\partial_{z} B_{x}$ across a shock. Further, we assume that the resistive contribution of the numerator in $D_{\mathrm{A} 1}$ is small, which is related to a small shock thickness. With these conditions, the variations of the numerator of Eq. (5) are bounded to small values compared to $\partial_{z} u_{z}$ across a shock. Then large values of $\partial_{z} u_{z}$ are restricted to the neighborhood of the singularities of Eq. (5), which are given by

$u_{z}= \pm \sqrt{c_{\mathrm{S}}^{2}+\widetilde{u}_{\mathrm{A}, x}^{2}}$.

Note that in resistive MHD, the singularities are usually removable and no infinite gradients occur. Although it is necessary to be close to such a singularity to obtain high values of $\partial_{z} u_{z}$, it is not a sufficient condition. The actual value of $\partial_{z} u_{z}$ depends on the limits of numerator and denominator. The velocity $u_{z}$ in Eq. (8) determines a characteristic velocity of wave propagation. If the flow velocity equals this characteristic velocity, shock waves can form due to the steepening of waves. For a uniform resistivity, the characteristic velocity is the magnetosonic velocity, as expected. Consider, e.g., the hydrodynamic limit where the magnetic field vanishes. Then $\widetilde{u}_{\mathrm{A}, x}=0$ in Eq. (5). An obstacle such as a sphere in the flow requires flow deflection away from the stagnation streamline to decelerate the flow, and consequently $D_{\mathrm{S}}>0$. If the initial flow velocity is supersonic, the flow has to pass the singularity in Eq. (5) during the deceleration. The resulting solution including the shock gives $D_{\mathrm{S}}=0$ ahead of the shock and $D_{\mathrm{S}}>0$ postshock (see, e.g., Nabert et al., 2013).

\section{Two-dimensional reconnection geometry}

We extend the previous analysis to investigate the velocity conditions for shocks in a two-dimensional diffusion region in the $x-z$ plane (Fig. 1). 


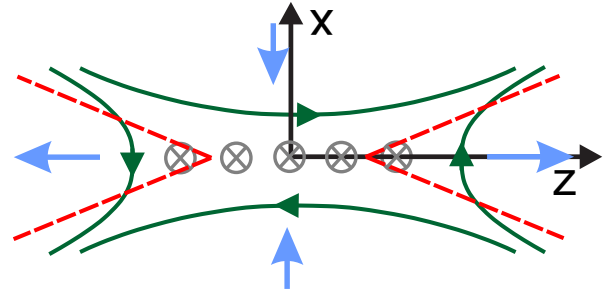

Figure 1. The current sheet of the diffusion region is represented by the circles with a cross. The plasma is reconnected at the $X$ point and is accelerated outward along the $z$ direction. Shock pairs can appear (dashed lines) for Petschek reconnection. Long arrows indicate magnetic field lines and short arrows the plasma inflow and outflow.

Thus, all components and variations with respect to $y$ are neglected. Then the stationary continuity equation reduces to

$u_{x} \partial_{x} \rho+\rho \partial_{x} u_{x}+u_{z} \partial_{z} \rho+\rho \partial_{z} u_{z}=0$.

Using the substitutions $\partial_{x} p=c_{\mathrm{S}}^{2} \partial_{x} \rho$ and $\partial_{z} p=c_{\mathrm{S}}^{2} \partial_{z} \rho$, the momentum Eq. (4) yields

$\rho u_{x} \partial_{x} u_{x}+\rho u_{z} \partial_{z} u_{x}+c_{\mathrm{S}}^{2} \partial_{x} \rho=B_{z} \partial_{z} B_{x}-B_{z} \partial_{x} B_{z}$

and

$\rho u_{x} \partial_{x} u_{z}+\rho u_{z} \partial_{z} u_{z}+c_{\mathrm{S}}^{2} \partial_{z} \rho=B_{x} \partial_{x} B_{z}-B_{x} \partial_{z} B_{x}$.

The $x$ component of the induction Eq. (2) gives

$u_{x} \partial_{z} B_{z}+B_{z} \partial_{z} u_{x}-u_{z} \partial_{z} B_{x}-B_{x} \partial_{z} u_{z}$

$=\partial_{z} \eta \partial_{x} B_{z}-\partial_{z} \eta \partial_{z} B_{x}+R_{1} \sqrt{\rho}$,

where $R_{1}:=\left(\eta \partial_{z} \partial_{x} B_{z}-\eta \partial_{z}^{2} B_{x}\right) / \rho^{0.5}$. To simplify the calculations, we assume that the resistivity depends on $z$, only. Then the $z$ component of the induction Eq. (2) is

$u_{z} \partial_{x} B_{x}+B_{x} \partial_{x} u_{z}-u_{x} \partial_{x} B_{z}-B_{z} \partial_{x} u_{x}=R_{2} \sqrt{\rho}$,

with $R_{2}:=\left(\eta \partial_{x} \partial_{z} B_{x}-\eta \partial_{x}^{2} B_{z}\right) / \rho^{0.5}$. As with the previous quasi one-dimensional considerations, expressions for the spatial variations of the MHD quantities are considered. Therefore, all partial derivatives of MHD quantities (e.g., $\left.\partial_{x} u_{z}, \partial_{z} B_{z}\right)$ are treated as variables. We choose a coordinate system with the $x$ axis along the normal direction of a possible shock. Note that Petschek's slow shocks are only approximately normal to the $z$ axis, so that the coordinate system $(x, z)$ has to be rotated until the $x$ axis matches the shock's normal direction. Then we can assume that variations of the MHD quantities with respect to the $z$ direction are usually minor compared to variations with respect to the $x$ direction. Note that one exception is $\partial_{x} B_{x}$, which vanishes approximately across a shock due to the divergence-free magnetic field if the $x$ direction is approximated as normal direction. Consequently, the system of Eqs. (9)-(13) is solved
Table 1. The coefficients in Eq. (15) using $\Delta u^{2}:=u_{x}^{2}-c_{\mathrm{S}}^{2}$ and $\Delta u_{z}:=u_{z}-\partial_{z} \eta$ to simplify the representation.

$$
\begin{aligned}
f_{1}= & u_{\mathrm{A}, z}\left(u_{\mathrm{A}, x}^{2} \Delta u^{2}+u_{x}^{2}\left(u_{\mathrm{A}, z}^{2}-\Delta u_{z} u_{z}\right)\right) \\
f_{2}= & u_{\mathrm{A}, z} c_{\mathrm{S}}^{2} u_{x} \Delta u_{z}-u_{\mathrm{A}, x}^{3} \Delta u^{2} \\
& -u_{\mathrm{A}, x}\left(u_{\mathrm{A}, z}^{2} u_{x}^{2}+\Delta u^{2} \Delta u_{z} u_{z}\right) \\
f_{3}= & \frac{\left(u_{x} u_{z}\left(c_{\mathrm{S}}^{2}\left(\partial_{z} \eta+u_{z}\right)-u_{x}^{2} \Delta u_{z}\right)\right)}{\rho^{0.5}} \\
f_{4}= & \frac{\left(u_{x}^{3} u_{\mathrm{A}}^{2}-u_{x} c_{\mathrm{S}}^{2} u_{\mathrm{A}, x}^{2}\right)}{\rho^{0.5}} \\
f_{5}= & \frac{\left(c_{\mathrm{S}}^{2} \Delta u_{z}\left(u_{\mathrm{A}, x} \Delta u^{2}+u_{\mathrm{A}, z} u_{x} u_{z}\right)\right)}{\rho} \\
f_{6}= & R_{2}\left(u_{x}^{3} u_{z}-\Delta u^{2} u_{x} \partial_{z} \eta\right)-R_{1}\left(u_{\mathrm{A}, x}^{2} \Delta u^{2}+u_{\mathrm{A}, z}^{2} u_{x}^{2}\right)
\end{aligned}
$$

with respect to $\partial_{x} u_{x}, \partial_{x} B_{z}, \partial_{z} B_{x}, \partial_{x} u_{z}$, and $\partial_{x} \rho$ which are the most significant variables at a shock. The solution for $\partial_{x} B_{z}$ is given by

$$
\partial_{x} B_{z}=\frac{D}{\left(\partial_{z} \eta-u_{z}\right)\left(u_{x}^{2}\left(u_{x}^{2}-c_{\mathrm{S}}^{2}-\kappa u_{\mathrm{A}}^{2}\right)+\kappa u_{\mathrm{A}, x}^{2} c_{\mathrm{S}}^{2}\right)},
$$

with $u_{\mathrm{A}}:=\left|\mathbf{u}_{\mathrm{A}}\right|$. The numerator is abbreviated by $D$, which can be written as

$D=f_{1} \partial_{z} u_{x}+f_{2} \partial_{z} u_{z}+f_{3} \partial_{x} B_{x}+f_{4} \partial_{z} B_{z}+f_{5} \partial_{z} \rho+f_{6}$,

with the coefficients displayed in Table 1.

Note that the solution for the chosen variables $\partial_{x} u_{x}, \partial_{x} B_{z}$, $\partial_{z} B_{x}, \partial_{x} u_{z}$, and $\partial_{x} \rho$ all provide the same denominator. Similarly to the analysis of the quasi one-dimensional flow, large values of $\partial_{x} B_{z}$ are related to the neighborhood of removable singularities in Eq. (14) because the terms with partial derivatives in the numerator are assumed to be small. The root of the denominator in Eq. (14) yields a relation for the critical flow velocity at the singularity which is given by

$u_{x}^{2}=\frac{1}{2}\left(c_{\mathrm{S}}^{2}+\kappa u_{\mathrm{A}}^{2}\right) \pm \sqrt{\kappa u_{\mathrm{A}, x}^{2} c_{\mathrm{S}}^{2}+\frac{1}{4}\left(c_{\mathrm{S}}^{2}+\kappa u_{\mathrm{A}}^{2}\right)^{2}}$.

If the condition in Eq. (16) is satisfied, strong variations in all the quantities along the $x$ direction can appear, a necessary condition to obtain a shock. For an incompressible plasma, the sound velocity approaches infinity $\left(c_{\mathrm{S}} \rightarrow \infty\right)$. Then $\left(u_{x}^{2}-c_{\mathrm{S}}^{2}-\kappa u_{\mathrm{A}}^{2}\right) \rightarrow\left(-c_{\mathrm{S}}^{2}\right)$, and the characteristic velocity of Eq. (14) gives the following nontrivial singularity conditions:

$u_{x}= \pm \tilde{u}_{\mathrm{A}, x}$.

If the resistivity is uniform, $\kappa \rightarrow 1$ and the characteristic velocity is the Alfvén velocity $u_{\mathrm{A}, x}$. In a nonuniform resistive 
plasma, a super-Alfvénic inflow with respect to the effective Alfvén velocity $\widetilde{u}_{\mathrm{A}, x}$ can cause a shock by passing the removable singularity.

To obtain a first insight into the effects of the inflow velocity conditions for shocks, we compare the limit of a uniform resistivity to a steep decreasing gradient of the resistivity along the $z$ direction. Such a steep decreasing gradient is expected for a locally enhanced resistivity on the right halfplane of the reconnection geometry in Fig. 1. The critical velocity for shocks of the inflow is determined by Eq. (17). For a uniform resistivity $\left(\partial_{z} \eta \rightarrow 0\right)$, the critical inflow velocity for shocks becomes the Alfvén velocity $u_{\mathrm{A}, x}$. Away from the $x$ axis and close to the $z$ axis, $u_{x} \leq u_{\mathrm{A}, x}$ due to the finite magnetic field $B_{x}$ and the vanishing velocity component $u_{x}$ (see Fig. 1). Far away from the $z$ axis, $u_{x}$ becomes the inflow velocity of the reconnection region and $u_{\mathrm{A}, x}$ vanishes. However, the $x$ axis is not strictly normal to the slow shock of Petschek reconnection. To correct this, we have to use the normal component of the magnetic field with respect to a possible shock instead of $B_{x}$, as discussed previously. Assuming a shock angle as proposed by the Petschek model for the slow shocks leads the inflow velocity to converge to the Alfvén velocity $u_{\mathrm{A}, x}$. However, the inflow remains below the critical velocity for Petschek's slow shocks for a uniform resistivity. A decreasing gradient of the resistivity, i.e., $\partial_{z} \eta<0$, leads to $\kappa<1$. As a consequence, the critical inflow velocity, determined by Eq. (17), becomes smaller. Then the inflow can exceed the effective Alfvén velocity and a shock can be triggered if the condition in Eq. (17) holds. The slow shocks in Petschek reconnection are such shocks. The limit $\partial_{z} \eta \rightarrow-\infty$ leads to $\kappa \rightarrow 0$, and thus, the effective Alfvén velocity $\widetilde{u}_{\mathrm{A}, x}$ vanishes. This leads to a critical flow velocity to obtain shocks of $u_{x}=0$, and waves cannot propagate against the upstream flow at the location of the gradient.

\section{Summary}

An approach to derive necessary conditions for shocks from the stationary resistive MHD equations was presented and applied to the slow shocks of Petschek reconnection. The approach treats terms with partial derivatives in the MHD equations as variables. The equations were solved with respect to terms with spatial derivatives which are normal to a possible shock. The resulting expressions are displayed by fractions, whereby the numerator contains the terms with spatial derivatives tangential to the possible shock. Across a shock, the variations of the MHD quantities are usually small compared to variations along the shock plane. Note that this assumes the curvature of a shock to be small, i.e., an approximately plane shock front. Therefore, the high values of the spatial derivatives of quantities normal to a shock, e.g., for the normal and tangential velocity components, require a root of the denominator in our expressions. This determines a necessary condition for a shock. The approach was introduced by a quasi one-dimensional situation with the magnetic field perpendicular to the flow velocity. This example is therefore limited to perpendicular shocks. Then a more general two-dimensional situation was considered. It was assumed that the resistivity profile is uniformly normal to a shock. The resulting necessary condition for a shock (Eq. 16) was discussed for the occurrence of subcritical slow shocks in Petschek reconnection depending on the resistivity profile. Therefore, the limit of an incompressible plasma was used which leads to Eq. (17). A gradient of the resistivity can significantly influence the necessary condition for shocks in the reconnection geometry. This is related to the slow shocks of Petschek reconnection. Although the approach was considered in the limit of a reconnection geometry, the necessary shock Eq. (16) holds for more general situations. Note that the critical velocity for shocks is related to a characteristic velocity of wave propagation. Therefore, the approach presented might be extended to consider changes of wave propagation in a nonuniform resistivity profile.

Acknowledgements. This work was financially supported by the German Ministerium für Wirtschaft und Technologie and the Deutsches Zentrum für Luft- und Raumfahrt under contracts 50OC1403, 50OC1402, and 50QW1101.

The topical editor, C. Owen, thanks H. Kucharek for help in evaluating this paper.

\section{References}

Achterberg, A.: A numerical study of steady-state shock acceleration, Astron. Astrophys., 174, 329-337, 1987.

Becker, P. A. and Kazanas, D.: Exact Expressions for the Critical Mach Numbers in the Two-Fluid Model of Cosmic-Ray-modified Shocks, Astrophys. J., 546, 429-446, doi:10.1086/318257, 2001.

Burgess, D. and Scholer, M.: Collisionless shocks in space plasmas: structure and accelerated particles, Cambridge University Press, Cambridge, 2015.

Ko, C. M., Chan, K. W., and Webb, G. M.: Cosmic-ray modified shocks with injection in the hydrodynamic approach - Part 1: Injection linear in the thermal pressure, J. Plasma Phys., 57, 677694, 1997.

Nabert, C., Glassmeier, K.-H., and Plaschke, F.: A new method for solving the MHD equations in the magnetosheath, Ann. Geophys., 31, 419-437, doi:10.5194/angeo-31-419-2013, 2013.

Parker, E. N.: Sweet's Mechanism for Merging Magnetic Fields in Conducting Fluids, J. Geophys. Res., 62, 509-520, doi:10.1029/JZ062i004p00509, 1957.

Petrinec, S. M. and Russell, C. T.: Hydrodynamic and MHD Equations across the Bow Shock and Along the Surfaces of Planetary Obstacles, Space Sci. Rev., 79, 757-791, doi:10.1023/A:1004938724300, 1997.

Petschek, H. E.: Magnetic Field Annihilation, NASA Special Publication, 50, 425, 1964. 
Scholer, M.: Undriven magnetic reconnection in an isolated current sheet, J. Geophys. Res., 94, 8805-8812, doi:10.1029/JA094iA07p08805, 1989.

Sweet, P. A.: The Neutral Point Theory of Solar Flares, in: Electromagnetic Phenomena in Cosmical Physics, edited by: Lehnert, B., 123 pp., Cambridge University Press, London, 1958.

Treumann, R. A.: Origin of resistivity in reconnection, Earth Planet. Space, 53, 453-462, doi:10.1186/BF03353256, 2001.

Ugai, M. and Tsuda, T.: Magnetic field-line reconnexion by localized enhancement of resistivity: I - Evolution in a compressible MHD fluid, J. Plasma Phys., 17, 337-356, doi:10.1017/S0022377800020663, 1977.
Yan, M., Lee, L. C., and Priest, E. R.: Fast magnetic reconnection with small shock angles, J. Geophys. Res., 97, 8277-8293, doi:10.1029/92JA00170, 1992.

Zita, E. J.: Analytic Origin of Advection of Magnetic Fields by Diffusivity Gradients, ArXiv e-prints, 2013. 